\title{
Capacidad Vesical, Edad, Peso, Talla y Superficie Corporal en Niños
}

\author{
Dr. Eugenio Rodríguez S. ${ }^{1}$ : Dr. Jorge Holzer M. 2; Dra. Ximana Triviño B. ${ }^{3}$; \\ Dr. Carlos Saieh A. 1 ; Dr. Federico Puga C. 1 \\ Bladder Capacity, Age, Weight, Height, and Body \\ Surface A rea
}

Thirty children between 4 year s 5 month and 13 years and half with normal intravenous urography, cistography, neurogical evaluation and urodinamical tests are presented. Simple and multiple correlations between bladder capacity: weight, height and surface area were investigated. The best linear relations were obtained for bladder capacity and height $(r=0.406 \mathrm{p}<0.05$ ). On the basis of this experience the authors propose a formula for the estimation of Bladder Capacity (BC) from height, where: Bladder capacity (ml) $=1.7 \times$ height $(\mathrm{cm})-0.4$ (Key words: Bladder, capacity. Wieght and bladder capacity).

La capacidad vesical en el niño ha sido relacionada por diversos autores con la edad, obteniendo una relación directamente proporcional entre estas dos variables. A partir de la capacidad vesical se han clasificado además, los trastornos del vaciamiento vesical,

En otra presentación, acerca de la Vejiga Inestable en la Infancia ${ }^{1}$, mostramos que la capacidad vesical no es buen parámetro en el diagnóstico de dichas disfunciones, lo que nos motivó para estudiar un grupo de niños con cistometría y estudios de radiología urológica normales, que consultaron por infección urinaria recurrente $y$ enuresis, incontinencia o ambos, tratando de relacionar la capacidad vesical con la edad, talla, peso y superficie corporal.

\section{MATERIAL Y METODO}

Entre los años 1982 y 1984 se estudiaron 30 niños que consultaron en la Unidad de Nefrourologia del Hospital Luis Calvo Mackenna, por infección urinaria recurrente, enuresis, incontinencia o ambos, a los cuales se midió la capacidad vesical máxima.

En todos se realizó examen neurológico, cistometria ${ }^{1}$, pielografía de eliminación y uretrocistografía, siendo informadas como normales.

De los 30 niños, 26 eran mujeres; sus edades fluctuaron entre 4 años 5 meses y 13 años 6 meses; todos estaban sin infección urinaria al menos un mes antes de la medición de la capaci-

1 Unidad de Nefrología, Hospital Luis Calvo Mackenna.

2 Insítuto de Neurología e investigaciones Cercbiates

3 Becada de Pediatría. Hospital Luis Calvo Mackenna. dad vesical y no habian recibido medicamento alguno durante ese mismo período.

La capacidad vesical se midió mediante una sonda Foley, a través de la cual se vació por completo la vejiga e instiló solución de $\mathrm{Na} \mathrm{Ci}$ al $9 \%$ en goteo lento, continuo a temperatura ambiente, hasta provocar sensación de micción inminente. La capacidad se definió como la suma del volumen de líquido emitido espontáneamente y del residuo que eventualmente quedase en la vejiga.

En el momento de medir la capacidad vesical, los niños fueron pesados y medidos. Se determinó la superficie corporal según el nomograma de W.M. Boothy $y$ R.B. Sandiford ${ }^{2}$.

Se estudiaron correlaciones simples entre capacidad vesical, talla, peso y superficie corporal $y$, con el objeto de elevar al máximo la eficiencia de la predicción, se realizó un análisis de correlación múltiple, tomando la capacidad vesical como "criterio" y la sunta ponderada de las variables talla-edad y peso edad respectivamente como "predictivas".

En la Tabla J se muestra la talla, peso, edad y superficie corporal media de los 30 pacientes estudiados.

\begin{tabular}{|c|c|c|c|c|}
\hline \multicolumn{5}{|c|}{$\begin{array}{c}\text { Tabla 1. } \\
\text { Parámetros Antroponétricos }\end{array}$} \\
\hline$(n=30)$ & $\begin{array}{l}\text { Talla } \\
(\mathrm{cm})\end{array}$ & $\begin{array}{l}\text { Peso } \\
(\mathrm{kg})\end{array}$ & $\begin{array}{l}\text { Edad } \\
\text { faños) }\end{array}$ & $\begin{array}{l}\text { Super- } \\
\text { ficie } \\
\left(\mathrm{m}^{2}\right)\end{array}$ \\
\hline Media & 128,3 & 27,8 & 8,8 & 0,91 \\
\hline $\pm D . E$. & 12,9 & 11,5 & 2.2 & 0.16 \\
\hline
\end{tabular}




\section{RESULTADOS}

La capacidad vesical máxima promedio fue de $217,4 \mathrm{ml}$ con \pm D.E. $53,8 \mathrm{mt}$.

La correlación de la capacidad vesical con la talla $(\mathrm{cm})$ tiene un $\mathrm{r}=0,406(\mathrm{p}<0,05)(\mathrm{Fig} .1)$.

La ecuación de regresión tiene la siguiente forma:

\section{Capacidad vesical $=1,7 \times$ talla $-0,4$}

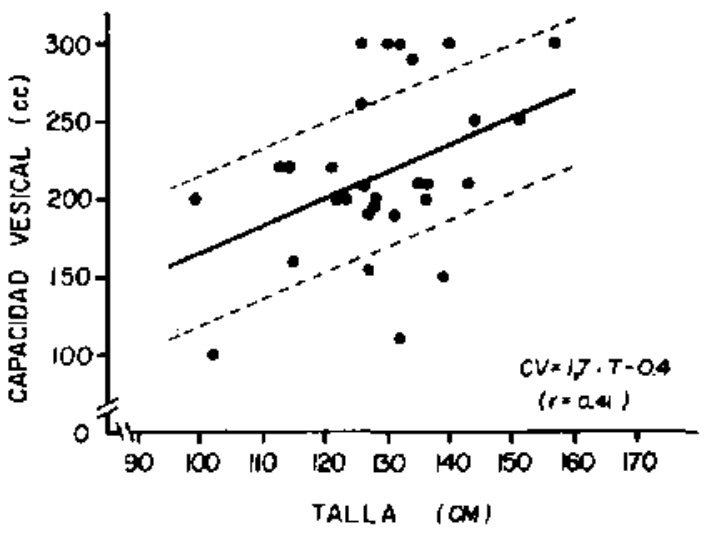

Figura 1.

Corrclación simple cntre capacidad resical y talla en 30 pacientes cistométricamente nomales

La correlación de la capacidad vesical con la superficie corporal $\left(\mathrm{m}^{2}\right)$ fue $\mathrm{r}=0,389(\mathrm{p}<0,05)$ (Fig. 2).

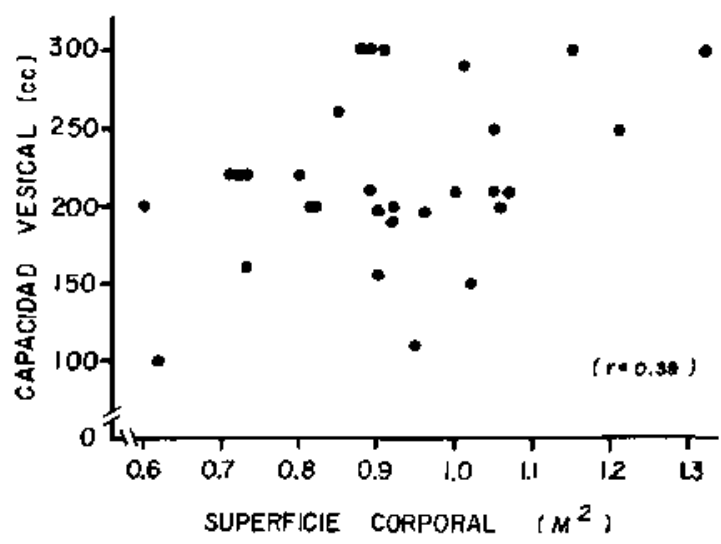

Figura 2.

Correlación simple entre capacidad vesical $y$ superticie corporal en 30 niños cistonétricamentc normales

La cortelación de la capacidad vesical con el peso $(\mathrm{Kg})$ tiene un $r=0,363$ con un $p$ n1o significativo.

La correlación de la capacidad vesical con tia edad (años) fue $\mathrm{r}=0,2 \mathrm{I} 7(\mathrm{p}>0,05)$.

La correlación múltiple entre capacidad vesical, talla y edad resultó con un $\mathrm{R}=0,47, y$ un error estándar de estimación para la predicción múltiple de 47,55. La ecuación de regresión múltiple tiene la forma siguiente:

Capacidad vesical $=3,2$ talla $\times 10,5$ edad -- 106.

La correlación entre capacidad vesical, talla y peso resultó $\mathrm{R}=0,408$.

La correlación entre la capacidad vesical, peso $y$ edad fue $\mathrm{R}=0,381$.

\section{DISCUSION}

La capacidad vesical media normal fue de $217,4 \mathrm{ml}$. \pm D.E. $53,8 \mathrm{ml}$, que significa que el $95 \%$ de los niños entre 4,5 y 13,5 años, tienen una capacidad vesical entre $109,4 \mathrm{ml}$ y $324 \mathrm{ml}$.

Al observar las correlaciones simples que efectuamos, la mejor fue entre la capacidad vesical y la talla corpural $(r=0,406)$.

Al tratar de aumentar la eficacia de la predicción con la correlación múltiple, esta mejora, pero en un porcentaje no mayor del $2 \%$, por to que ratificamos que la mejor correlación práctica para el cálculo de la capacidad vesical es aplicando la recta de regresión: Capacidad vesical = $1,7 \times$ talla $-0,4$.

Jay discusión en la literatura con respecto a la relación entre capacidad resical y edad. En concordancia con algunas publicaciones, que demuestran que entre 4,5 y 14 años de edad, no hay correlación entre capacidad vesical y edad, nuestra experiencia demostró un coeficiente de correlación no válido $(\mathrm{p}>0,05)$ entre estas variables 10

Dada la dispersión de los valores normales, clasificar los trastornos vesicales funcionales basándose en la capacidad vesical, no nos parece correcto, a pesar de las publicaciones extranjeras al respecto ${ }^{3-9}$. Es posible concluir que la capacidad vesical en los niños, es muy variable y no guarda relación signifjcativa con la edad entre 4.5 y 13,5 años. La mejor relación ocurre entre la capacidad vesical y la talla corporal en estas edades.

\section{RESUMEN}

En 30 nif̂́os de 4 años 5 meses a 13 años 6 meses de edad con antecedentes de infección urinaria e incontinencia, enuresis o ambas, se midió la capacidad vesical intentando relacionarla con el peso, la estatura y la superficie corporal. La mejor relaciốn ocurrió con la estatura $(r=$ 
$0,406 ; \mathrm{p}<0.05)$, que con el peso $(\mathrm{r}=0,389 ; \mathrm{p}<$

$0.05)$ y la edad $(r=0,217 ; p>0.05)$.

La ecuación que relaciona la capacidad vesical con la estatura, está representada en la fórmula:

Capacidad Vesical $(\mathrm{mi})=1,7 \times$ talla $(\mathrm{cm})$ $-0,4$.

\section{REFERENCIAS}

1 Rodriguez E., Holzer J., Triviño X., y CaL: La vejiga inestable en la infancia. Rey. Chil. Pediatr. 56(1); en Prensa, 1985.

2 Boorhby W.M., Sandiford R.B.: Nomographic charts for the calculation of the metabolic rate by the gasometer method. Boston. MSJ. 18S: 337, 1921.

${ }^{3}$ Baver S. et al: The unstable bladder of childhood Urologic clinics of North America. 2: 321, 1980.
${ }^{4}$ Muellner. S.R.: Development of urinary control in children. Some aspects of the cause and treatment of primary enenuresis. J.A.M.A. 172: 1256, 1960.

${ }^{5}$ Berger R.M. et al: Bladder capacity (ounces) equals age (years) plus 2 predicts normal bladder capacity and aids in diagnosis of abnortmal yoiding patterns. 1. Urol. 129:347, 1983 .

${ }^{6}$ Allen T.D., Bright T.C.: Urodynamic patterns in children with dys functional voiding problems. J. Urol. 119:24, 1978.

${ }^{7}$ Blaivas J.G., Labib K.G., Bauer S. B. er, al: Changing concepts in the urodynamic evaluation of childran. J. Urol. 117: 778, 1977.

${ }^{8}$ Firlit C.F., Smey P., King L.R., Micturition: Urodinamic flow studites in children. J. Urol. 111: 250, 1978.

${ }^{9}$ Storfield B.: Functional bladder capacity in enuretic and nonenuretic children. J. Pediatr. 70:777, 1977.

10 Muellner S.R.: Development of urinary control in children. Some aspects of the cause and treatment of primary enuresis. J.A.M.A. 172: 1256, 1960. 